\title{
Fetoplacental mosaicism: potential implications for false-positive and false-negative noninvasive prenatal screening results
}

\author{
Francesca R. Grati, PhD¹, Francesca Malvestiti, PhD¹, Jose C.P.B. Ferreira, MD, PhD², Komal Bajaj, MD², \\ Elisa Gaetani, BSc ${ }^{1}$, Cristina Agrati, BSc ${ }^{1}$, Beatrice Grimi, BSc ${ }^{1}$, Francesca Dulcetti, PhD ${ }^{1}$, \\ Anna M. Ruggeri, BSc${ }^{1}$, Simona De Toffol, $\mathrm{PhD}^{1}$, Federico Maggi, PhD ${ }^{1}$, Ronald Wapner, $\mathrm{MD}^{3}$, \\ Susan Gross, $\mathrm{MD}^{2}$ and Giuseppe Simoni, $\mathrm{PhD}^{1}$
}

Purpose: Noninvasive prenatal screening for fetal aneuploidy analyzes cell-free fetal DNA circulating in the maternal plasma. Because cellfree fetal DNA is mainly of placental trophoblast origin, false-positive and false-negative findings may result from placental mosaicism. The aim of this study was to calculate the potential contribution of placental mosaicism in discordant results of noninvasive prenatal screening.

Methods: We performed a retrospective audit of 52,673 chorionic villus samples in which cytogenetic analysis of the cytotrophoblast (direct) and villus mesenchyme (culture) was performed, which was followed by confirmatory amniocentesis in chorionic villi mosaic cases. Using cases in which cytogenetic discordance between cytotrophoblast and amniotic fluid samples was identified, we calculated the potential contribution of cell line-specific mosaicism to falsepositive and false-negative results of noninvasive prenatal screening.
Results: The false-positive rate, secondary to the presence of abnormal cell line with common trisomies in cytotrophoblast and normal amniotic fluid, ranged from $1 / 1,065$ to $1 / 3,931$ at $10 \%$ and $100 \%$ mosaicism, respectively; the false-negative rate was calculated from cases of true fetal mosaicism, in which a mosaic cell line was absent in cytotrophoblast and present in the fetus; this occurred in $1 / 107$ cases.

Conclusion: Despite exciting advances, underlying biologic mechanisms will never allow $100 \%$ sensitivity or specificity.

Genet Med advance online publication 13 February 2014

Key Words: cell-free placental DNA; false-negative rate; falsepositive rate; fetoplacental mosaicism; noninvasive prenatal screening

\section{INTRODUCTION}

Although the utility of analyzing cell-free DNA (cfDNA) in maternal circulation as a means of ascertaining important information about a pregnancy was recognized almost 2 decades ago, ${ }^{1}$ it is only recently that noninvasive prenatal screening (NIPS) for fetal aneuploidy using cffDNA has been available commercially. 2,3 Although NIPS is described as a "fetal" screening test and authors refer to "cell-free fetal DNA" (cffDNA) in the context of the test, ${ }^{4,5}$ this is a misnomer because the DNA actually originates from apoptosis of placental cytotrophoblast and syncytiotrophoblast cells. ${ }^{6-8}$

Studies to date in which NIPS was performed demonstrate high sensitivity and specificity for detection of the common aneuploidies (trisomies 13,18, and 21) with a rapid rise in the uptake of NIPS worldwide. However, concerns regarding the paucity of scientific data, performance characteristics, and consequent clinical utility are now being raised ${ }^{9}$ and, as with all screening tests, false-positive (FP) and false-negative (FN) results occur. ${ }^{10-13}$ There are various explanations for FP and FN results, with fetoplacental mosaicism, in which the cytotrophoblast but not the fetus (FP) - and vice versa (FN) - contains the aneuploid cell line, being a primary potential mechanism.
Determining the frequency of confined placental mosaicism (CPM) and true fetal mosaicism (TFM) requires that, in the analysis of villus samples, karyotyping of both the cytotrophoblast and the mesenchyme is performed together with the confirmatory analysis on amniotic fluid (AF) in case of mosaicism detected in chorionic villi. Although cytogenetic analysis of the cytotrophoblast layer from which NIPS DNA is derived is no longer conducted in many laboratories, we continue to assess both placental tissues (cytotrophoblast and mesenchyme) on all chorionic villus sampling (CVS) studies. Using our extensive database, our aim was to calculate the potential contribution of fetoplacental mosaicism to NIPS FP and $\mathrm{FN}$ results.

\section{MATERIALS AND METHODS}

\section{Study conduct and laboratory procedures}

After approval of the TOMA Laboratory Institutional Review Board, a retrospective cytogenetic audit of 52,673 consecutive prenatal diagnoses of CVS analyzed by the TOMA laboratory in a 13-year period (May 2000-May 2013) was performed.

Cases underwent similar procedures using consistent evaluation criteria and procedures in agreement with the Italian and

${ }^{1}$ Research \& Development, Cytogenetics, Molecular Cytogenetics and Molecular Biology, TOMA Advanced Biomedical Assays, S.p.A., Busto Arsizio (VA), Italy; ${ }^{2}$ Department of Obstetrics and Women's Health, Albert Einstein College of Medicine, Bronx, New York, USA; ${ }^{3}$ Department of Obstetrics and Gynecology, Columbia University Medical Center, New York, New York, USA. Correspondence: Francesca R. Grati (fgrati@tomalab.com) 
European guidelines, as previously reported. ${ }^{14,15}$ In the direct preparation technique, metaphases were obtained "directly" from spontaneously dividing in vivo cytotrophoblasts using the protocol originally developed by Simoni et al. in $1983 .{ }^{16}$ Mesenchymal core analysis was performed on cultured cells, as previously described. ${ }^{17} \mathrm{~A}$ total of at least 16 chorionic villi metaphases were routinely counted and paired. When an abnormal direct preparation was identified along with a normal female mesenchyme culture, a total of 40 metaphases from two cultures were counted to minimize the risk of maternal cell contamination.

Mosaicism was defined as a normal cell line plus either (i) the presence of at least two cells with the same trisomy or structural rearrangement or (ii) three cells showing the same monosomy in at least one placental tissue (cytotrophoblast and/or mesenchyme).$^{18}$ In mosaic CVS cases, a confirmatory amniocentesis was always recommended. Karyotyping from AF was performed using the in situ technique, and we analyzed 20 metaphases from a minimum of 10 colonies taken from at least two in situ cultures. TFM was defined as the presence of at least two colonies derived from two different cultures showing the same abnormality in AF as observed in CVS. A nonmosaic fetoplacental discrepancy was the homogeneous presence of an abnormal cell line in only one of the analyzed tissues. ${ }^{12}$ Mosaicism was classified according to the distribution of the abnormal cell line and is described in Table $1 .{ }^{18}$

\section{Data analysis}

To calculate the potential contribution of mosaicism to FP and FN NIPS, we evaluated mosaics in which the cytotrophoblast was cytogenetically discrepant from the fetus. Specifically, we evaluated CPM type 1, in which the chromosomal abnormality is only in the cytotrophoblast and not in the mesenchyme and AF; CPM type 3, in which the chromosomal abnormality is found both in the cytotrophoblast and the mesenchymal core and not in AF; and TFM type 5, in which aneuploid cells are absent in the cytotrophoblast but present in the villus mesenchyme and the fetus. CPM types 1 and 3 would contribute to FP NIPS results and TFM type 5 to FN results.

We selected only mosaics involving chromosome imbalances targeted or potentially identifiable by NIPS: trisomies 13,18 , and 21, monosomy X (MX), 47,+i(13q), 47,+i(21q), 47,XXX/ $\mathrm{XXY} / \mathrm{XYY}, 46, \mathrm{X}, \operatorname{del}(\mathrm{Xq}), 46, \mathrm{X}, \mathrm{i}(\mathrm{Xq})$, mosaic 45,X/47,XXX and $47,+\mathrm{i}(18 \mathrm{p})$. In this calculation, $47,+\mathrm{i}(13 \mathrm{q})$ and $47,+\mathrm{i}(21 \mathrm{q})$ were counted as T13 and T21, respectively (Table 2).

The potential contribution to the FP rate for each chromosome abnormality was calculated by dividing the number of CPM 1 and 3 cases by the number of normal cases: number of $\mathrm{FP}(\mathrm{CPM} 1+\mathrm{CPM} 3) /$ true negative cases + FP(CPM1 + CPM3). The contribution to the FN rate was calculated by dividing the number of TFM type 5 by the number of all abnormal cases: number of FN(TFM5)/true positive cases + FN(TFM5). Data are further presented stratified by the percentage of abnormal cells in the cytotrophoblast to illustrate the effect and the role of percentage mosaicism on FP rates. The total FN rate is calculated as 1 minus sensitivity and the FP rate as 1 minus specificity.

\section{RESULTS}

Of the 52,673 cases, 308 had CPM type 1, 90 had CPM type 3, and 51 had TFM type 5; among these, 45 of CPM1 (14.6\%), 13 of CPM3 (14.4\%), and 25 of TFM5 (29.4\%) cases involved the common trisomies 13,18 , and 21 .

\section{FP rate}

The potential contribution to the FP rate from CPM type 1 or 3 with trisomic cell lines quantified between $\geq 10 \%$ through $100 \%$ is reported in Table 2. Even using a conservative assumption that a high percentage of mosaicism $\geq 70 \%$ abnormal cells would be required to produce sufficient fetal fraction to generate a "positive" by NIPS, the FP rate for the common trisomies $(13,18$, and 21$)$ would be $0.033 \%$ or 1 in 3,006 cases reported as normal ( $95 \%$ confidence interval (CI): $1 / 1,877-1 / 4,813)$; for common trisomies and monosomy $\mathrm{X}$, the $\mathrm{FP}$ rate would be $0.08 \%$, which is equivalent to 1 in 1,243 cases reported as normal (95\% CI: $1 / 917-1 / 1,687)$; and for all targeted or potentially identifiable chromosome abnormalities, the FP rate would be $0.091 \%$, which is equivalent to 1 in 1,105 cases reported as normal (95\% CI: 1/829-1/1,474).

\section{FN rate}

FN cases involving T13, 18, and 21 are projected to occur in 1/107 karyotypes reported as abnormal (95\% CI: 1/65-1/176); for T13, 18 , and 21 and MX, it will occur in 1/68 abnormal karyotypes (95\% CI: 1/46-1/99); for all targeted or potentially identifiable chromosome abnormalities (including structural anomalies

Table 1 Schematic representation of the types of mosaicism and the expected NIPS results

\begin{tabular}{lllll} 
Type of mosaic & $\begin{array}{c}\text { Cytotrophoblast (direct } \\
\text { preparation or short-term culture) }\end{array}$ & $\begin{array}{c}\text { Mesenchyme } \\
\text { (long-term culture) }\end{array}$ & Amniocytes & Expected NIPS result \\
\hline CPM1 & Abnormal & Normal & Normal & FP \\
CPM2 & Normal & Abnormal & Normal & TN \\
CPM3 & Abnormal & Abnormal & Abnormal & FP \\
TFM4 & Abnormal & Normal & Abnormal & TP* \\
TFM5 & Normal & Abnormal & Abn \\
TFM6 & Abnormal & Abnormal & Abnal & TP* \\
\hline
\end{tabular}

CPM, confined placental mosaicism; FN, false negative; FP, false positive; NIPS, noninvasive prenatal screening; TFM, true fetal mosaicism; TN, true negative; TP*, true positive assuming a consistent percentage of the mosaic abnormal cell line in cytotrophoblast. 


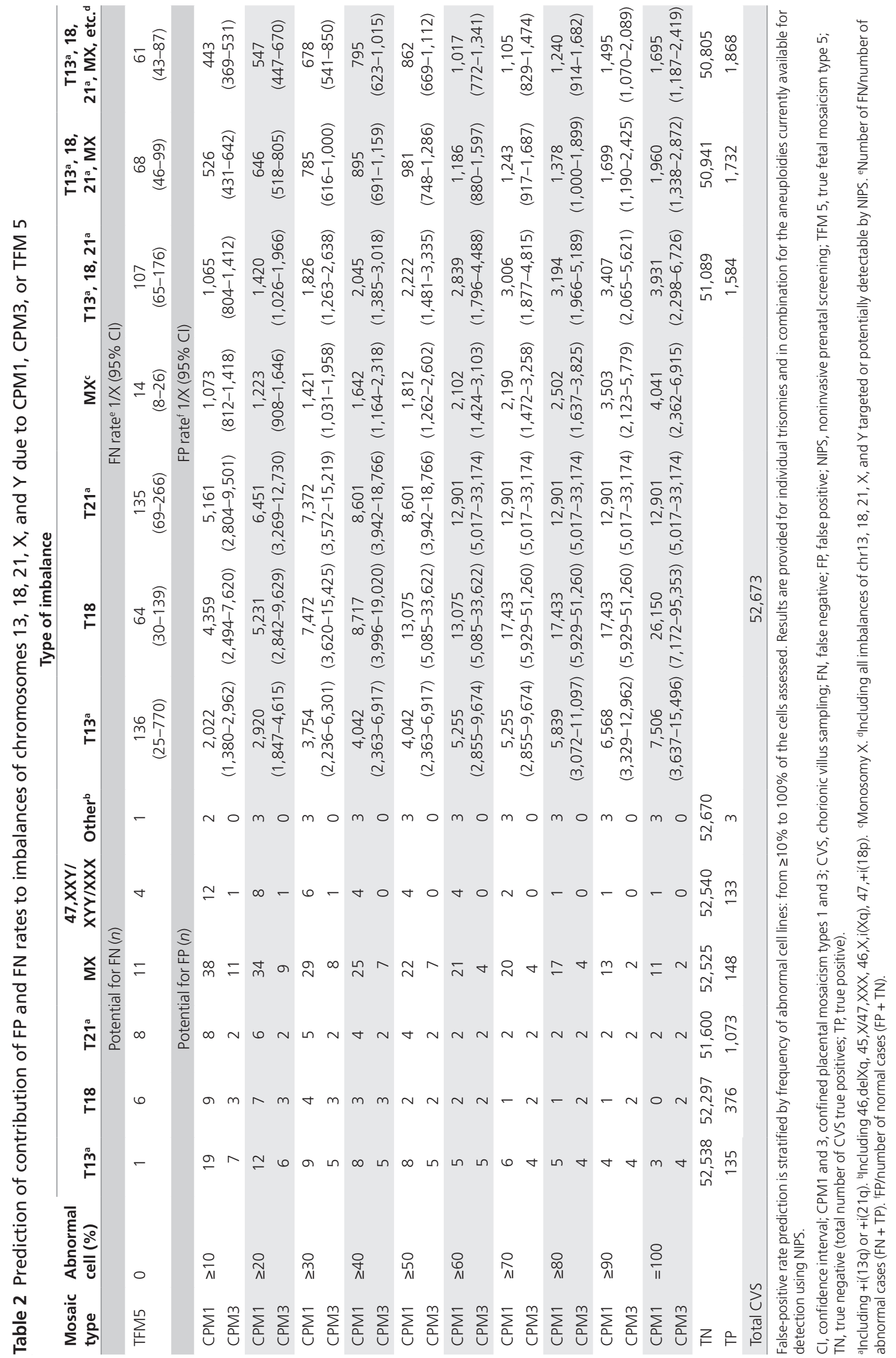


involving these chromosomes), it will occur in 1/61 abnormal karyotypes (95\% CI: 1/43-1/87). The rate of FN, stratified by type of aneuploidy, is as follows: for T13, 1/136 (95\% CI: 1/251/770); for T18, $1 / 64$ (95\% CI: 1/30-1/139); for T21, 1/135 (95\% CI: 1/69-1/266); and for MX, 1/14 (95\% CI: 1/8-1/26; Table 2).

\section{Conclusion}

NIPS has been a long-awaited addition to the tools available for the care of pregnant women. Although the sensitivity and the specificity of NIPS have been reported to approach $99 \%$, several issues, such as performance characteristics of the different assays, remain, which are detailed in the study by Morain et al. ${ }^{9}$ Even the lay press ${ }^{19}$ has raised flags that FP and FN are expected in "screening" tests, but with marketing to both patients and doctors that stresses high sensitivity and specificity, there is confusion, and pitfalls abound with respect to informed consent. Because this screening test is being used on a more frequent basis in a larger population, providers must be able to inform patients regarding its limitations, including the risk of a FP or FN screen result.

CPM is a well-known biologic phenomenon that is likely to result from mitotic or meiotic nondisjunction errors and trisomy rescue. Recently published case reports have suggested this to be a mechanism underlying some false/discordant NIPS results. ${ }^{12,13}$ Our analysis, based on a large cytogenetic data set that incorporates both the cytotrophoblast and the mesenchymal core, allows the prediction of the potential contribution of CPM and TFM to the rates of FP and FN NIPS results, respectively. However, it is important to note that verification of these predicted results would require prospective data to be obtained.

Patients undergoing CVS for cytogenetic analysis should be counseled that the tissue being sampled is not fetal and that mosaic conditions can occur. We propose that the term cell-free fetal DNA, "cffDNA," is more appropriate for sequences derived from $\mathrm{AF}^{20}$ Because NIPS is primarily a "placental" assay, this term should be changed to cell-free placental DNA, "cfpDNA." As a result, patients will be made aware that there is a small chance of a discrepancy that may require further confirmation through amniocentesis. Of note, the test is indeed accurate for the targeted abnormalities, because, in the vast majority of cases, the cytotrophoblast accurately reflects the fetal chromosomal status. However, comparable with CVS, a "positive" NIPS test may reflect only the cytotrophoblast and not the fetal karyotype and therefore must always be confirmed by invasive testing. Similarly, physicians must include in NIPS counseling that the outer placental cellular layer may be normal, but the fetus may still be affected and, therefore, a negative NIPS may be relatively reassuring due to a generally high negative predictive value; however, NIPS cannot completely rule out an abnormal fetal karyotype, even for the limited disorders being tested.

Discrepancies between NIPS and fetal results may fail to be explained by extensive cytogenetic analysis of term placenta. Syncytial sprouts, present from the early stage of pregnancy, are considered to be a morphological manifestation of villus growth and represent the first step in the development of lateral villi. ${ }^{21}$ The number and size of these sprouts increase steadily during the first 3 months of pregnancy, paralleling the increase in serum level of gonadotropins. ${ }^{22}$ At the end of 20 weeks of gestation, the cytotrophoblast completely disappears, ${ }^{23}$ and this might explain such unexplained discrepancies.

The cytotrophoblast is the mitotically active cell of the villi, and cytogenetic results are obtained without in vitro culture, analyzing only spontaneous metaphases. Thus, the cytogenetic results used in this study reflect the actual constitution of the outer placental cellular layer. Although FP results are dependent on the percentage of mosaicism, the fetal fraction of the maternal plasma, and the type of NIPS (single-nucleotide-poymorphism-based or counting-based) technology, the estimated FN rate due to TFM type 5 can be considered a reliable estimation because it is independent of the technical and physiological variants. In the future, deeper sequencing might enable the identification of lower-level mosaics, and our data provide a projection of the increase in the FP rate attributed to CPM as the sensitivity of NIPS technologies increases.

There are other reported reasons for discrepancies between fetal karyotype and NIPS results, including a vanishing twin or a co-twin's demise, ${ }^{24}$ nonmosaic maternal chromosome abnormality, ${ }^{25}$ and maternal metastatic disease. ${ }^{26}$ Particularly in the case of FN, low fetal fraction ${ }^{27}$ may play a key role. This study quantifies the role of CPM/TFM and does not address these other potential etiologies.

Published studies report variable FP rates, which are summarized as $1 / 384$ for T21 and $1 / 500$ for T18. ${ }^{28} \mathrm{FN}$ rates (one minus the detection rate) were summarized as $1 / 200$ for T21 and $1 / 63$ for T18. ${ }^{28}$ Our results support the hypothesis that CPM types 1 and 3 do contribute to the FP rate of NIPS and that TFM type 5 may be a major contributor to the suboptimal sensitivity.

Although our study indicates that $\mathrm{FP}$ and $\mathrm{FN}$ results may very well be the result of placental biology and not a failure in the actual test platform, it also demonstrates that although we can expect further technological and bioinformatic breakthroughs in the future, underlying placental-fetal genetic mechanisms will never allow $100 \%$ sensitivity.

\section{DISCLOSURE}

F. M. and G.S. are owners of TOMA Advanced Biomedical Assays S.p.A., a prenatal diagnosis laboratory mainly involved in cytogenetic analysis on prenatal samples. R.W. is the principal investigator on grants funded by Ariosa Diagnostics and Natera. All of this funding goes to Columbia University. He has no personal funding from these companies or personal conflicts. The other authors declare no conflict of interest.

\section{REFERENCES}

1. Lo YM, Corbetta N, Chamberlain PF, et al. Presence of fetal DNA in maternal plasma and serum. Lancet 1997;350:485-7.

2. Bianchi DW, Platt LD, Goldberg JD, Abuhamad AZ, Sehnert AJ, Rava RP; MatErnal BLood IS Source to Accurately diagnose fetal aneuploidy (MELISSA) Study Group. Genome-wide fetal aneuploidy detection by maternal plasma DNA sequencing. Obstet Gynecol 2012;119:890-901. 
3. Chiu RW, Akolekar R, Zheng YW, et al. Non-invasive prenatal assessment of trisomy 21 by multiplexed maternal plasma DNA sequencing: large scale validity study. BMJ 2011;342:C7401.

4. Verweij EJ, Jacobsson B, van Scheltema PA, et al. European Non-Invasive Trisomy Evaluation (EU-NITE) study: a multicenter prospective cohort study for noninvasive fetal trisomy 21 testing. Prenat Diagn 2013;22:1-6.

5. Samuel A, Bonanno C, Oliphant A, Batey A, Wright JD. Fraction of cell-free fetal DNA in the maternal serum as a predictor of abnormal placental invasion-a pilot study. Prenat Diagn 2013;33:1-4.

6. Tjoa ML, Cindrova-Davies T, Spasic-Boskovic O, Bianchi DW, Burton GJ. Trophoblastic oxidative stress and the release of cell-free feto-placental DNA. Am J Pathol 2006;169:400-4.

7. Flori E, Doray B, Gautier E, et al. Circulating cell-free fetal DNA in maternal serum appears to originate from cyto- and syncytio-trophoblastic cells. Case report. Hum Reprod 2004;19:723-4.

8. Faas BH, de Ligt J, Janssen I, et al. Non-invasive prenatal diagnosis of fetal aneuploidies using massively parallel sequencing-by-ligation and evidence that cell-free fetal DNA in the maternal plasma originates from cytotrophoblastic cells. Expert Opin Biol Ther 2012;12 Suppl 1:S19-26.

9. Morain S, Greene MF, Mello MM. A new era in noninvasive prenatal testing. NEngl J Med 2013;369:499-501.

10. Mennuti MT, Cherry AM, Morrissette JJ, Dugoff L. Is it time to sound an alarm about false-positive cell-free DNA testing for fetal aneuploidy? Am J Obstet Gynecol 2013;209:415-9.

11. Mozersky J, Mennuti MT. Cell-free fetal DNA testing: who is driving implementation? Genet Med 2013;15:433-4.

12. Gao $Y$, Stejskal D, Jiang F, Wang W. A T18 false negative result by NIPT in a XXX, T18 case due to placental mosaicism. Ultrasound Obstet Gyneco/ 2013. doi:10.1002/uog.13240.

13. Pan M, Li FT, Li Y, et al. Discordant results between fetal karyotyping and noninvasive prenatal testing by maternal plasma sequencing in a case of uniparental disomy 21 due to trisomic rescue. Prenat Diagn 2013;33:598-601.

14. Grati FR, Grimi B, Frascoli G, et al. Confirmation of mosaicism and uniparental disomy in amniocytes, after detection of mosaic chromosome abnormalities in chorionic villi. Eur J Hum Genet 2006;14:282-8.

15. Grati FR, Malvestiti F, Grimi B, et al. QF-PCR as a substitute for karyotyping of cytotrophoblast for the analysis of chorionic villi: advantages and limitations from a cytogenetic retrospective audit of 44,727 first-trimester prenatal diagnoses. Prenat Diagn 2013;33:502-8.

16. Simoni G, Brambati B, Danesino C, et al. Efficient direct chromosome analyses and enzyme determinations from chorionic villi samples in the first trimester of pregnancy. Hum Genet 1983;63:349-57.

17. Verma RS, Babu A. Human Chromosomes Principles and Techniques. McGrawHill, Inc, 1995:24-6 (protocol 2.16).

18. McKinlay Gardner RJ, Sutherland GR, Shaffer LG: Chromosome Abnormalities and Genetic Counseling (Oxford Monographs on Medical Genetics), 4th edn, chapter 27. Oxford University Press: New York, 2012:439-85.

19. Tough calls on prenatal tests. The Wall Street Journal. http://online.wsj.com/ article/SB10001424127887324883604578398791568615644. Accessed 23 August 2013.

20. Hui L, Bianchi DW. Cell-free fetal nucleic acids in amniotic fluid. Hum Reprod Update 2011;17:362-71.

21. Bennington JL. The development and tructure of the placenta. In: Pathology of the Placenta (Major Problems in Pathology), vol. 8. Sauders: London, 1978: $1-37$.

22. Ciblis LA. Growth of the placenta villi in the first trimester. J Reprod Med 1968;1:377-387.

23. Kaufmann P. Development and differentiation of the human placental villous tree. Bibl Anat 1982;22:29-39.

24. Futch T, Spinosa J, Bhatt S, de Feo E, Rava RP, Sehnert AJ. Initial clinical laboratory experience in noninvasive prenatal testing for fetal aneuploidy from maternal plasma DNA samples. Prenat Diagn 2013;33:569-74.

25. Yao $\mathrm{H}$, Zhang $\mathrm{L}$, Zhang $\mathrm{H}$, et al. Noninvasive prenatal genetic testing for fetal aneuploidy detects maternal trisomy X. Prenat Diagn 2012;32: 1114-6.

26. Osborne CM, Hardisty E, Devers P, et al. Discordant noninvasive prenatal testing results in a patient subsequently diagnosed with metastatic disease. Prenat Diagn 2013;33:609-11.

27. Canick JA, Palomaki GE, Kloza EM, Lambert-Messerlian GM, Haddow JE. The impact of maternal plasma DNA fetal fraction on next generation sequencing tests for common fetal aneuploidies. Prenat Diagn 2013;33:667-74.

28. Benn P, Cuckle H, Pergament E. Non-invasive prenatal testing for aneuploidy: current status and future prospects. Ultrasound Obstet Gynecol 2013;42: 15-33. 